\title{
BMJ
}

\section{Aspirin "resistance" and risk of cardiovascular morbidity: systematic review and meta-analysis}

\author{
George Krasopoulos, cardiovascular surgery fellow, ${ }^{1}$ Stephanie J Brister, associate professor, ${ }^{1}$ \\ W Scott Beattie, R Fraser Elliot chair in cardiac anaesthesia, ${ }^{2}$ Michael R Buchanan, professor ${ }^{3}$
}

${ }^{1}$ University Health Network, Division of Cardiovascular Surgery, Toronto General Hospital

2University Health Network Department of Anaesthesiology, Toronto General Hospital

${ }^{3}$ McMaster University, Department of Pathology and Molecular Medicine, Hamilton, ON, Canada L8S 4L8

Correspondence to: M R Buchanan mbuchan@mcmaster.ca

doi:10.1136/bmj.39430.529549.BE

\section{ABSTRACT}

Objective To determine if there is a relation between aspirin "resistance" and clinical outcomes in patients with cardiovascular disease.

Design Systematic review and meta-analysis.

Data source Electronic literature search without language restrictions of four databases and hand search of bibliographies for other relevant articles.

Review methods Inclusion criteria included a test for platelet responsiveness and clinical outcomes. Aspirin resistance was assessed, using a variety of platelet function assays.

Results 20 studies totalling 2930 patients with cardiovascular disease were identified. Most studies used aspirin regimens, ranging from 75-325 mg daily, and six studies included adjunct antiplatelet therapy. Compliance was confirmed directly in 14 studies and by telephone or interviews in three. Information was insufficient to assess compliance in three studies. Overall, 810 patients (28\%) were classified as aspirin resistant. A cardiovascular related event occurred in $41 \%$ of patients (odds ratio $3.85,95 \%$ confidence interval 3.08 to 4.80 ), death in $5.7 \%(5.99,2.28$ to 15.72$)$, and an acute coronary syndrome in $39.4 \%$ (4.06, 2.96 to 5.56). Aspirin resistant patients did not benefit from other antiplatelet treatment. Conclusion Patients who are resistant to aspirin are at a greater risk of clinically important cardiovascular morbidity long term than patients who are sensitive to aspirin.

\section{INTRODUCTION}

There is no debate that long term aspirin use attenuates the risks of myocardial infarction, stroke, and vascular related deaths in patients with cardiovascular disease, ${ }^{1}$ but a significant number of patients prescribed aspirin as antithrombotic therapy have major adverse vascular related events each year. ${ }^{2}$ Consequently other antiplatelet agents in addition to aspirin have been prescribed for certain patients. ${ }^{\text {w16-w } 20}$

The major controversy about aspirin therapy is why particular patients do not benefit from such therapy and how they might be identified. It has been suggested that some patients require a higher dose of aspirin than is normally recommended to achieve the expected antiplatelet effect-for example, inhibition of platelet function or inhibition of platelet thromboxane $\mathrm{A}_{2}$ synthesis..$^{3-6 w 10}$ It is unclear whether these patients simply receive too low an aspirin dose, are not compliant, have differing abilities to absorb aspirin, or have an underlying genetic disposition that renders aspirin ineffective. ${ }^{27-11}$ Such patients have been labelled aspirin "resistant"- that is, their platelets are not affected in the same way or are affected differently from the platelets of those who seem to benefit from aspirin therapy (aspirin "sensitive" patients with no subsequent adverse cardiovascular event). ${ }^{12-17 w 1 \text { w4-w9 }}$ w11-w13 Little consistency exists about which measure should be used to identify patients who seem resistant

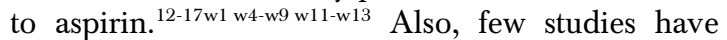
assessed the effect of aspirin resistance on clinically important outcomes.

We systematically reviewed studies of aspirin resistance and its effect on adverse cardiovascular outcomes. We hypothesised that aspirin resistance is real and is clinically relevant - that is, it significantly affects the risk of cardiovascular, cerebrovascular, and vascular related events. ${ }^{11 \mathrm{w} 6}$

\section{METHODS}

Using no language restrictions we systematically searched for studies on antiplatelet therapy from 1966 to the present through Medline, Embase, CHINAHL, and the Cochrane Controlled Trials Registry. We used the search terms "acetylsalicylic acid", "aspirin", "antiplatelet”, "antiplatelets", "platelets", "drug resistance", "aspirin resistance", and "platelet resistance". The initial search identified 36573 papers. When we refined our search to aspirin resistance the number of papers was reduced to 320 , and 210 remained when we further refined the search to aspirin resistance and clinical outcome. An expert hand searched and reviewed citations within these 210 key papers. We reviewed 268 abstracts for evidence of data related to aspirin resistance and its relation to clinical outcome. We excluded papers that did not contain a direct measure of platelet function or any data on clinical outcome in relation to aspirin resistance. Overall we independently reviewed 57 papers in detail.

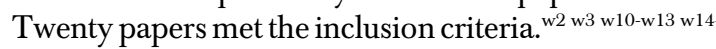
${ }^{\text {w20 }}$ Figure 1 shows the flow of papers through the trial. 
Potential relevant studies identified by

electronic and manual searches $(n=320)$

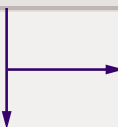

Trial excluded by screening of

titles and abstracts $(n=268)$

Potential studies retrieved for

complete evaluation $(n=57)$

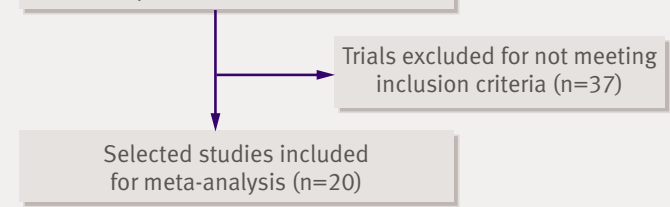

Fig 1 Flow of papers through review process

We each reviewed and tabulated the data from every paper. When possible we approached the original authors to verify our interpretation of their data. Data that had been confirmed for accuracy were then analysed using Review Manager 4.2.8 for metaanalysis.

\section{Inclusion and exclusion criteria}

We included studies that met the following criteria: (a) participants were receiving aspirin therapy as an antithrombotic; (b) participants were classified prospectively as aspirin sensitive or aspirin resistant before the ascertainment of any clinical outcome, or were grouped on the basis of clinical outcome and then classified for aspirin status (we considered patients to be aspirin sensitive if their platelets responded as expected to aspirin treatment, and platelet function, however measured, was inhibited, and we considered patients to be aspirin resistant if their agonist induced platelet response was not inhibited by aspirin as expected); (c) there was adequate allocation concealment such that investigators were blinded to the patients' aspirin sensitive and aspirin resistant status; (d) a measure of prospective clinical outcome was used in both groups of patients; and (e) patients receiving other antiplatelet treatment were also included. (The expectation was that there would be an attenuation of the rate of any adverse effects associated with aspirin resistance in the presence of a second platelet inhibitor, given the perceived rationale for prescribing the second antiplatelet agent. Alternatively, if no attenuation of any adverse events occurred in the presence of a second platelet inhibitor, this could possibly reflect a lack of any benefit of the second platelet inhibitor.)

The 37 studies that we excluded did not assess an agonist induced platelet response to ascertain patients' aspirin resistance status, or lacked an assessment of the relation between aspirin resistance and clinical morbidity or mortality. This included any study that measured only thromboxane $\mathrm{A}_{2}$ (thromboxane $\mathrm{B}_{2}$ ) levels, ${ }^{8}$ because inhibition of thromboxane $B_{2}$ synthesis could simply reflect a measure of patient compliance.
We considered several platelet function assays acceptable for inclusion: whole blood platelet function tests, such as the platelet function analyser 100 test (Dade Behring, Deerfield, IL, USA); light transmission platelet rich plasma platelet function tests; and bleeding time as a function of platelet haemostasis. The platelet function analyser 100 test is thought to measure shear stress induced platelet activation - that is, adherence to and occlusion of a membrane coated with a platelet agonist in vitro. Light transmission aggregometry measures the increase in light transmitted through a suspension of platelet rich plasma exposed to various agonists. The Surgicutt II (ITC Commercial Group, Edison, NJ, USA) is thought to measure platelet dependent haemostasis. None of these assays were weighted differently from one another from the perspective of one test being better than another in identifying aspirin resistance, particularly as no consensus on this has been reached. ${ }^{18}$

\section{Outcome measures}

The papers reported on four primary outcomes: any cardiovascular or cerebrovascular event (for example, fatal or non-fatal myocardial infarction, stroke, vascular related event); death; acute coronary syndromes (for example, non-ST segment elevation myocardial infarction, ST elevation myocardial infarction, unstable angina); and failure of vascular interventions (vein failure or reocclusion, revascularisation, and vascular restenosis).

\section{Data collection and analysis}

We collected personal and clinical outcome data from the included studies. Before analysis we confirmed the accuracy of the data. We rechecked any disparate data and corrected accordingly. When possible we contacted one of the original authors by email to verify the accuracy of their data and the interpretation of their results by the current investigators. Nurses fluent in Chinese or Czech translated the two foreign language studies. ${ }^{\mathrm{w} 4}$ w20

\section{Statistical analysis}

We present the results as odds ratios with 95\% confidence intervals. We considered a two tailed $\mathrm{P}$ value of less than 0.05 as significant. Calculations were done using the fixed effects model. Heterogeneity was assessed using the $Q$ test (a search being initiated by a heterogeneity $>50 \%$ ). Finally, we determined the robustness of our findings by removing the most positive studies one at a time. Planned sensitivity analyses were assessment of the platelet function assays, dose of aspirin, and the inclusion of another platelet inhibitor. We used a computer assisted S-plus/ $\mathrm{R}$ function to fit meta-analytic mixed random and fixed effects models to do a metaregression analysis of any dose response. ${ }^{19}$ Potential publication bias was assessed by a funnel plot, and we used an Egger's regression test to assess any dose related effect. ${ }^{20} \mathrm{We}$ used the checklist recommended by the meta-analysis of observational studies in epidemiology group. ${ }^{21}$ 


\section{Table 1| Characteristics of included studies}

\begin{tabular}{|c|c|c|c|c|c|c|c|c|c|}
\hline Study & $\begin{array}{l}\text { Design; } \\
\text { quality* }\end{array}$ & $\begin{array}{c}\text { Patient } \\
\text { population }\end{array}$ & $\begin{array}{c}\text { No of } \\
\text { patients }\end{array}$ & $\begin{array}{l}\text { Aspirin } \\
(\mathrm{mg} / \mathrm{d})\end{array}$ & $\begin{array}{c}\text { Adjunct } \\
\text { antiplatelet } \\
\text { therapy }\end{array}$ & Compliance & $\begin{array}{l}\text { No of patients } \\
\text { aspirin resistant; } \\
\text { No of patients } \\
\text { aspirin sensitive }\end{array}$ & Assay & $\begin{array}{l}\text { Outcome } \\
\text { measures }\end{array}$ \\
\hline Borna et al $2005^{\mathrm{w} 1}$ & Cohort; A & $\begin{array}{l}\text { Acute coronary } \\
\text { syndrome }\end{array}$ & 64 & $75-320$ & - & Verbal & $20 ; 44$ & $\begin{array}{l}\text { Platelet function } \\
\text { analyser } 100\end{array}$ & $\begin{array}{l}\text { Acute coronary } \\
\text { syndrome }\end{array}$ \\
\hline Cotter et al $2004^{\mathrm{w} 2}$ & Cohort; A & $\begin{array}{c}\text { Post } \\
\text { myocardial } \\
\text { infarction }\end{array}$ & 61 & 100 & - & $\begin{array}{l}\text { Baseline: aspirin in } \\
\text { hospital }\end{array}$ & $9 ; 52$ & $\begin{array}{c}\text { Platelet } \\
\text { aggregation† }\end{array}$ & $\begin{array}{c}\text { Cardiovascular or } \\
\text { cerebrovascular } \\
\text { events }\end{array}$ \\
\hline $\begin{array}{l}\text { Grundmann et al } \\
2003^{w 3}\end{array}$ & Cohort; A & $\begin{array}{l}\text { Cardiovascular } \\
\text { disease or } \\
\text { stroke }\end{array}$ & 53 & 100 & - & $?$ & $12 ; 41$ & $\begin{array}{l}\text { Platelet function } \\
\text { analyser } 100\end{array}$ & New strokes \\
\hline Stejskal et al ${ }^{\mathrm{w} 4}$ & Cohort; A & $\begin{array}{l}\text { Acute coronary } \\
\text { syndrome }\end{array}$ & 103 & 100 & - & $?$ & $73 ; 30$ & $\begin{array}{c}\text { Platelet } \\
\text { aggregation } †\end{array}$ & $\begin{array}{c}\text { Acute coronary } \\
\text { syndrome }\end{array}$ \\
\hline $\begin{array}{l}\text { Andersen et al } \\
2003^{w 5}\end{array}$ & $\begin{array}{l}\text { Prospective } \\
\text { randomised } \\
\text { controlled; A }\end{array}$ & $\begin{array}{c}\text { Post } \\
\text { myocardial } \\
\text { infarction }\end{array}$ & 71 & 160 & - & Repeat measures & $25 ; 46$ & $\begin{array}{l}\text { Platelet function } \\
\text { analyser } 100\end{array}$ & $\begin{array}{c}\text { Cardiovascular or } \\
\text { cerebrovascular } \\
\text { events } \neq\end{array}$ \\
\hline $\begin{array}{l}\text { Buchanan et al } \\
2000^{w 6}\end{array}$ & $\begin{array}{c}\text { Multicentre } \\
\text { prospective } \\
\text { descriptive; } \\
\text { A }\end{array}$ & $\begin{array}{l}\text { Post coronary } \\
\text { artery bypass } \\
\text { grafting }\end{array}$ & 289 & 325 & - & $\begin{array}{l}\text { Baseline: aspirin in } \\
\text { hospital }\end{array}$ & $158 ; 131$ & $\begin{array}{c}\text { Serum thromboxane } \\
\mathrm{B}_{2} \text { level, bleeding } \\
\text { time } \S\end{array}$ & $\begin{array}{c}\text { Cardiovascular or } \\
\text { cerebrovascular } \\
\text { events }\end{array}$ \\
\hline Gum et al $2003^{w 7}$ & Cohort; A & $\begin{array}{l}\text { Cardiovascular } \\
\text { disease }\end{array}$ & 326 & 325 & - & Verbal & $17 ; 309$ & $\begin{array}{c}\text { Platelet } \\
\text { aggregation† }\end{array}$ & $\begin{array}{c}\text { Cardiovascular or } \\
\text { cerebrovascular } \\
\text { events }\end{array}$ \\
\hline $\begin{array}{l}\text { Hobikoglu et al } \\
2005^{\text {w8 }}\end{array}$ & Cohort; A & $\begin{array}{l}\text { Acute coronary } \\
\text { syndrome }\end{array}$ & 204 & $100-300$ & - & $\begin{array}{l}\text { Baseline: aspirin in } \\
\text { hospital }\end{array}$ & $69 ; 135$ & $\begin{array}{l}\text { Platelet function } \\
\text { analyser } 100\end{array}$ & $\begin{array}{c}\text { Cardiovascular or } \\
\text { cerebrovascular } \\
\text { events }\end{array}$ \\
\hline McCabe et al $2005^{\mathrm{w} 9}$ & $\begin{array}{c}\text { Multicentre } \\
\text { cohort; A }\end{array}$ & $\begin{array}{c}\text { Cerebrovascu- } \\
\text { lar }\end{array}$ & 47 & $75-300$ & - & Verbal & $22 ; 25$ & $\begin{array}{l}\text { Platelet function } \\
\text { analyser } 100\end{array}$ & $\begin{array}{c}\text { Cardiovascular or } \\
\text { cerebrovascular } \\
\text { events }\end{array}$ \\
\hline Meuller et al $1997^{\mathrm{w} 10}$ & Cohort; D & $\begin{array}{l}\text { Peripheral } \\
\text { vascular } \\
\text { disease }\end{array}$ & 96 & 100 & - & $\begin{array}{l}\text { Baseline: aspirin in } \\
\text { hospital }\end{array}$ & $41 ; 55$ & $\begin{array}{l}\text { Whole blood } \\
\text { aggregometry }\end{array}$ & $\begin{array}{c}\text { Vascular } \\
\text { occlusion or } \\
\text { deterioration }\end{array}$ \\
\hline Poston et al $2006^{\mathrm{w} 11}$ & Cohort; A & $\begin{array}{c}\text { Post coronary } \\
\text { artery bypass } \\
\text { grafting }\end{array}$ & 225 & 325 & - & $\begin{array}{l}\text { Baseline: aspirin in } \\
\text { hospital }\end{array}$ & $49 ; 176$ & $\begin{array}{l}\text { TEG, whole blood } \\
\text { aggregometry, } \\
\text { thromboxane } \mathrm{B}_{2}\end{array}$ & Graft failure \\
\hline Yilmaz et al $2005^{\mathrm{w} 12}$ & $\begin{array}{c}\text { Prospective } \\
\text { case- } \\
\text { control; A }\end{array}$ & $\begin{array}{c}\text { Post coronary } \\
\text { artery bypass } \\
\text { grafting }\end{array}$ & 28 & $100-325$ & - & $\begin{array}{l}\text { Baseline: aspirin in } \\
\text { hospital }\end{array}$ & $8 ; 20$ & $\begin{array}{l}\text { Platelet function } \\
\text { analyser } 100\end{array}$ & Graft failure \\
\hline Grotemeyer et al ${ }^{\mathrm{w} 13}$ & Cohort; A & Stroke & 174 & 1500 & - & $\begin{array}{l}\text { Baseline: aspirin in } \\
\text { hospital }\end{array}$ & $60 ; 114$ & Platelet adhesion & $\begin{array}{c}\text { Cardiovascular or } \\
\text { cerebrovascular } \\
\text { events }\end{array}$ \\
\hline $\begin{array}{l}\text { Parmukcu et al } \\
2007^{\mathrm{w} 14}\end{array}$ & Cohort; D & $\begin{array}{c}\text { Coronary } \\
\text { artery disease }\end{array}$ & 234 & $100-300$ & - & $?$ & $52 ; 182$ & $\begin{array}{l}\text { Platelet function } \\
\text { analyser } 100\end{array}$ & $\begin{array}{c}\text { Cardiovascular or } \\
\text { cerebrovascular } \\
\text { events }\end{array}$ \\
\hline $\begin{array}{l}\text { Berrouschot et al } \\
2006^{\text {w15 }}\end{array}$ & Cohort; A & Stroke & 240 & 300 & $\begin{array}{c}\text { Aspirin plus } \\
\text { clopidogrel for } \\
\text { aspirin resistant } \\
\text { patients }\end{array}$ & $\begin{array}{l}\text { Baseline: aspirin in } \\
\text { hospital }\end{array}$ & $30 ; 210$ & $\begin{array}{c}\text { Platelet } \\
\text { aggregation } †\end{array}$ & New strokes \\
\hline Chen et al $2005^{\mathrm{w} 16}$ & Cohort; A & $\begin{array}{l}\text { Percutaneous } \\
\text { coronary } \\
\text { intervention }\end{array}$ & 151 & $80-320$ & $\begin{array}{l}\text { Aspirin plus } \\
\text { clopidogrel }\end{array}$ & $\begin{array}{c}\text { Baseline: aspirin in } \\
\text { hospital }\end{array}$ & $29 ; 122$ & Ultegra & $\begin{array}{l}\text { Acute coronary } \\
\text { syndrome }\end{array}$ \\
\hline Chen et al $2004^{\mathrm{w} 17}$ & Cohort; A & $\begin{array}{l}\text { Percutaneous } \\
\text { coronary } \\
\text { intervention }\end{array}$ & 117 & $80-320$ & $\begin{array}{l}\text { Aspirin plus } \\
\text { clopidogrel }\end{array}$ & $\begin{array}{c}\text { Baseline: aspirin in } \\
\text { hospital }\end{array}$ & $22 ; 95$ & VerifyNow & $\begin{array}{l}\text { Acute coronary } \\
\text { syndrome }\end{array}$ \\
\hline Faraday et al $2004^{\mathrm{w} 18}$ & Cohort; A & $\begin{array}{l}\text { Acute coronary } \\
\text { syndrome }\end{array}$ & 30 & 325 & $\begin{array}{l}\text { Aspirin plus } \\
\text { clopidogrel and } \\
\text { tirofiban }\end{array}$ & $\begin{array}{l}\text { Baseline: aspirin in } \\
\text { hospital }\end{array}$ & $2 ; 28$ & $\begin{array}{c}\text { Platelet } \\
\text { aggregation } †\end{array}$ & $\begin{array}{l}\text { Acute coronary } \\
\text { syndrome }\end{array}$ \\
\hline Gurbel et al $2003^{\mathrm{w} 19}$ & Cohort; A & $\begin{array}{l}\text { Percutaneous } \\
\text { coronary } \\
\text { intervention }\end{array}$ & 191 & $81-325$ & $\begin{array}{l}\text { Aspirin plus } \\
\text { clopidogrel and } \\
\text { glycoprotein } \\
\text { Ilb/IIla }\end{array}$ & $\begin{array}{c}\text { Baseline: aspirin in } \\
\text { hospital }\end{array}$ & $45 ; 146$ & TEG & $\begin{array}{c}\text { Cardiovascular or } \\
\text { cerebrovascular } \\
\text { events }\end{array}$ \\
\hline Zhang et al $2005^{\mathrm{w} 20}$ & Cohort; D & $\begin{array}{l}\text { Percutaneous } \\
\text { coronary } \\
\text { intervention }\end{array}$ & 256 & 100 & Plus clopidogrel & $\begin{array}{l}\text { Baseline: aspirin in } \\
\text { hospital }\end{array}$ & $67 ; 189$ & $\begin{array}{c}\text { Platelet } \\
\text { aggregation† }\end{array}$ & $\begin{array}{l}\text { Acute coronary } \\
\text { syndrome }\end{array}$ \\
\hline
\end{tabular}

*Risk of bias: from A (low) to D (insufficient information for assessment of bias).

†Laboratory specific platelet aggregation assays, using stimuli such as arachidonic acid, adenosine diphosphate, or collagen.

$\ddagger$ Fatal or non-fatal events.

$\S$ Bleeding time using Surgicutt II. 


\begin{tabular}{|c|c|c|c|c|c|c|c|c|}
\hline \multirow{2}{*}{$\begin{array}{l}\text { Study } \\
\text { Grotemeyer et al }\left.\right|^{\text {w13 }}\end{array}$} & \multirow{2}{*}{$\begin{array}{c}\text { No with event/ } \\
\text { No of aspirin } \\
\text { resistant } \\
\text { patients } \\
24 / 60\end{array}$} & \multirow{2}{*}{$\begin{array}{c}\text { No with event/ } \\
\text { No of aspirin } \\
\text { sensitive } \\
\text { patients } \\
5 / 114\end{array}$} & \multicolumn{3}{|c|}{$\begin{array}{c}\text { Odds ratio (fixed) } \\
(95 \% \mathrm{Cl})\end{array}$} & & \multirow{2}{*}{$\begin{array}{c}\text { Weight } \\
\text { (\%) } \\
2.65\end{array}$} & \multirow{2}{*}{$\begin{array}{c}\begin{array}{c}\text { Odds ratio (fixed) } \\
\text { (95\% Cl) }\end{array} \\
14.53(5.16 \text { to 40.89) }\end{array}$} \\
\hline & & & & & & $\longrightarrow$ & & \\
\hline McCabe et al ${ }^{\text {w9 }}$ & $2 / 22$ & $1 / / 25$ & & & & $\longrightarrow$ & 1.09 & 2.40 (0.20 to 28.45$)$ \\
\hline Mueller et al ${ }^{\text {w10 }}$ & $8 / 41$ & $0 / 55$ & & & & & 0.44 & $28.16(1.57$ to 503.85$)$ \\
\hline Buchanan et al ${ }^{\mathrm{w} 6}$ & $15 / 158$ & $9 / 131$ & & & $\rightarrow$ & & 11.41 & 1.42 (0.60 to 3.36$)$ \\
\hline Andersen et $\mathrm{al}^{\mathrm{w5}}$ & $9 / 25$ & $11 / 46$ & & & $\square$ & & 6.35 & $1.79(0.62$ to 5.17$)$ \\
\hline Grundmann et al ${ }^{\text {W3 }}$ & $12 / 12$ & $23 / 41$ & & & & $\longrightarrow$ & 0.55 & 19.68 (1.09 to 354.69$)$ \\
\hline Gum et al ${ }^{\mathrm{w7}}$ & $4 / 17$ & $30 / 309$ & & & & & 3.06 & 2.86 (0.88 to 9.33$)$ \\
\hline Chen et al ${ }^{\mathrm{w} 16}$ & $19 / 29$ & $47 / 122$ & & & _ & & 7.97 & $3.03(1.30$ to 7.08$)$ \\
\hline Cotter et al ${ }^{\mathrm{w} 2}$ & $1 / 9$ & $3 / 52$ & & & $\square$ & $\rightarrow$ & 1.01 & 2.04 (0.19 to 22.13$)$ \\
\hline Faraday et al ${ }^{\mathrm{w} 18}$ & $1 / 2$ & $1 / 28$ & & & & $\rightarrow$ & 0.09 & 27.00 (0.89 to 821.79$)$ \\
\hline Stejskal et al ${ }^{\text {w4 }}$ & $61 / 73$ & $1 / 30$ & & & & 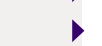 & 0.30 & 142.42 (18.28 to 1188.69$)$ \\
\hline Borna et al ${ }^{\mathrm{w} 1}$ & $17 / 20$ & $15 / 44$ & & & & $\longrightarrow$ & 1.80 & $10.96(2.77$ to 43.40$)$ \\
\hline Chen et al ${ }^{\mathrm{w} 17}$ & $10 / 22$ & $17 / 95$ & & & & $\rightarrow$ & 4.47 & $3.82(1.42$ to 10.29$)$ \\
\hline Gurbel et al ${ }^{\mathrm{w} 19}$ & $27 / 45$ & $11 / 146$ & & & & $\rightarrow$ & 2.66 & 18.41 (7.82 to 43.35$)$ \\
\hline Hobikoglu et al ${ }^{\text {w8 }}$ & $42 / 69$ & $62 / 135$ & & & 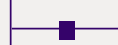 & & 21.02 & 1.83 (1.02 to 3.30$)$ \\
\hline Yilmaz et al ${ }^{\mathrm{w} 12}$ & $7 / 8$ & $7 / 20$ & & & 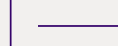 & $\rightarrow$ & 0.64 & $13.00(1.32$ to 128.11$)$ \\
\hline Zhang et al ${ }^{\mathrm{w} 20}$ & $41 / 67$ & $67 / 189$ & & & 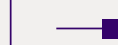 & & 17.43 & 2.87 (1.62 to 5.10$)$ \\
\hline Berrouschot et al ${ }^{\text {w15 }}$ & $1 / 30$ & $8 / 210$ & & & & & 2.48 & $0.87(0.11$ to 7.22$)$ \\
\hline Poston et al ${ }^{\mathrm{w} 11}$ & $7 / 49$ & $9 / 146$ & & & $\longrightarrow$ & & 4.97 & 2.54 (0.89 to 7.22$)$ \\
\hline Pamukcu et al ${ }^{\mathrm{w} 14}$ & $8 / 52$ & $20 / 182$ & & & $\longrightarrow$ & & 9.63 & $1.47(0.61$ to 3.57$)$ \\
\hline Total $(95 \% \mathrm{Cl})$ & 810 & 2120 & & & & & 100.00 & 3.85 (3.08 to 4.80$)$ \\
\hline \multicolumn{9}{|l|}{ Test for heterogeneity: } \\
\hline \multicolumn{2}{|c|}{$\chi^{2}=60.69, d f=19, P<0.001, I^{2}=68.7 \%$} & 0.1 & 0.2 & 0.5 & 2 & 10 & & \\
\hline \multicolumn{2}{|c|}{ Test for overall effect: $z=11.93, P<0.001$} & Dec & creased & & & sed risk & & \\
\hline
\end{tabular}

Fig 2 Risk of any cardiovascular event in aspirin resistant patients

\section{RESULTS}

The systematic search identified 20 studies, totalling 2930 patients with cardiovascular disease (17 cohort studies; one multicentre, descriptive study; and two case-control studies). The studies included only prospectively collected data on a variety of vascular related diseases: previous stroke (414 patients), acute coronary syndrome $(\mathrm{n}=401)$, myocardial infarction $(\mathrm{n}=132)$, coronary arterial bypass grafting $(n=542)$, percutaneous coronary intervention $(\mathrm{n}=715)$, stable cardiovascular disease $(\mathrm{n}=760)$, and peripheral vascular disease ( $\mathrm{n}=96$; table 1 ). All but three of the studies were assessed as of A quality (low risk of bias). In the three studies incomplete reporting prevented the ascertainment of risk of underlying bias. ${ }^{\text {w10 } 14 \text { w20 }}$ These studies were small, however, and their inclusion or exclusion was unlikely to significantly affect overall reporting. No studies were of a prospective, randomised clinical design (with or without placebo controls).

Thirteen studies reported on aspirin only as antiplatelet therapy, with daily doses ranging from 75$325 \mathrm{mg}$; one study used a daily dose of $500 \mathrm{mg}$ three times daily, and six studies included a loading dose of clopidogrel (Plavix) or another antiplatelet inhibitor tirofiban hydrochloride, or both, as adjunct therapy. ${ }^{\text {w15- }}$ w20 Compliance was assessed by the primary study investigator in 17 of the 20 studies.
A variety of assays were used to assess patients' aspirin status. These included measuring serum thromboxane $\mathrm{A}_{2}$ in relation to platelet haemostasis, ${ }^{\text {w6 }}$ a platelet or collagen adhesion assay, ${ }^{\mathrm{w} 13}$ platelet rich plasma aggregometry, ${ }^{\mathrm{w} 2 \mathrm{w} 4 \mathrm{w} 7 \mathrm{w} 15 \mathrm{w} 18 \mathrm{w} 20}$ whole blood platelet aggregometry, such as the platelet function analyser 100 test, ${ }^{\text {w1 }}$ w3 w5 w8 w9 w12 w14 or some combina-

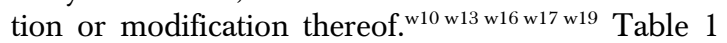
summarises the characteristics of the included studies.

Overall, 2120 of the 2930 patients were classified as aspirin sensitive and the remaining $810(28 \%)$ as aspirin resistant. Aspirin resistance was less prevalent in men than in women and higher in patients with previous renal impairment $(\mathrm{P}<0.001$ and $\mathrm{P}<0.03)$. No other differences in the clinical and personal characteristics between aspirin resistant and aspirin sensitive patients were apparent (table 2).

All aspirin resistant patients, regardless of underlying clinical symptoms, were at a greater risk of death, acute coronary syndrome, failure in vascular intervention, or a new cerebrovascular event (table 3): 39\% of aspirin resistant patients compared with $16 \%$ of aspirin sensitive patients had a cardiovascular event (odds ratio 3.85, 95\% confidence interval 3.08 to 4.80 ; $\mathrm{P}<0.001$, fig 2). The odds ratios for increased acute coronary syndrome, graft failure, or a new cerebrovascular event were 4.06, 4.35, and 3.78. Moreover, the odds ratio for increased mortality in aspirin resistant patients was 5.99 (2.28 to $15.72 ; \mathrm{P}<0.003)$. 
In the results for cardiovascular outcome the overall heterogeneity was $68.3 \%$. Most of the heterogeneity $(50.3 \%)$ was contributed to by the eight studies that used the whole blood platelet function analyser 100 test; 33\% of those patients were identified as aspirin resistant $(2.94,1.88$ to $4.55 ; \mathrm{P}=0.00001)$. In contrast, heterogeneity was $0 \%$ in the seven studies that used a platelet rich plasma aggregation assay; only $16 \%$ of patients were identified as aspirin resistant, and these patients had a higher odds ratio $(3.85,2.5$ to 5.88; $\mathrm{P}<0.001)$.

Data were insufficient to assess the effect of the whole blood aggregometry assays TEG (Haemoscope, Niles, IL, USA) and VerifyNow (Accumetrics, San Diego, CA, USA) and bleeding time assays on overall heterogeneity.

In the planned sensitivity analysis no evidence was found of a dose-response relation between aspirin resistance and any cardiovascular outcome in those patients who received aspirin alone or who received a second antiplatelet treatment (table 3, fig 3). Thus the overall odds ratio was 3.28 (95\% confidence interval 2.39 to $4.49, \mathrm{P}<0.001)$, but no relation was found between dose and adverse outcome measures $\left(\mathrm{R}^{2}=0.0046\right.$; fig 4). Moreover, concomitant therapy with clopidogrel or tirofiban (an inhibitor of platelet glycoprotein IIb/IIIa), or both, provided no benefit to those patients identified as aspirin resistant (aspirin and clopidogrel, 3.06, 1.99 to 4.70; aspirin alone, 2.52, 1.79 to 3.56 ; fig 3).

A funnel plot indicated an absence of published data of small studies and negative results - that is, no studies reported no adverse effect in aspirin resistant patients (lower left quadrant in figure 5). Little evidence was, however, found of publication bias using the classic funnel plot (fig 5) or a Macaskill ${ }^{22}$ modification (not shown).

\section{DISCUSSION}

Meta-analysis showed that patients who are "resistant" to aspirin are at greater risk of clinically important adverse cardiovascular events, regardless of the assay used to measure aspirin resistance. Not only did aspirin resistance have an effect on clinical outcome but this risk was not ameliorated by currently used adjunct antiplatelet therapies.

Patients who were classified as aspirin resistant were at about a fourfold increased risk of non-fatal and fatal cardiovascular, cerebrovascular, or vascular events while taking aspirin than their aspirin sensitive counterparts. This risk can be generalised to a wide variety of patient populations with cardiovascular or cerebrovascular disease.

\section{Putting our study into context}

The concept of aspirin resistance has been debated since the 1980s, ${ }^{249-11 w 1321}$ but discussions in the recent literature have focused on reasons why aspirin resistance is probably a misnomer, and to which little clinical relevance can be attached. ${ }^{211}$ More recently, however, interest has been renewed in aspirin resistance, which has focused primarily on identifying the platelet related assay that best reflects the phenomenon. ${ }^{2111823-26}$ To date this issue has not been resolved. Few studies have tackled the matter of aspirin resistance and its impact on clinical outcome. Given the lack of resolution of a suitable assay and the limited size, number, and applicability of individual studies, we carried out a systematic review and meta-analysis of all available studies from 1960 to the present to understand better the relation between aspirin resistance and

Table 2 | Characteristics of included patients

\begin{tabular}{|c|c|c|c|c|c|c|c|}
\hline Characteristic & $\begin{array}{l}\text { Aspirin } \\
\text { resistant } \\
\text { patients }\end{array}$ & $\begin{array}{l}\text { Aspirin } \\
\text { sensitive } \\
\text { patients }\end{array}$ & $\begin{array}{l}\text { Heterogeneity } \\
\text { (\%) }\end{array}$ & Odds ratio $(95 \% \mathrm{Cl})$ & $P$ value & $\begin{array}{l}\text { No of studies } \\
\quad(n=20)\end{array}$ & $\begin{array}{l}\text { No of patients } \\
\quad(n=2930)\end{array}$ \\
\hline $\begin{array}{l}\text { Mean (SD) age } \\
\text { (years) }\end{array}$ & $62.2(10.1)$ & $62.7(11.3)$ & 0 & $0.51(-0.82$ to 1.84$)$ & 0.45 & 7 & 1554 \\
\hline No (\%) of men & $(71.4)$ & $(79.5)$ & - & 2.08 (1.54 to 2.82$)$ & 0.001 & 7 & 1554 \\
\hline Hypertension & $202 / 397$ & $507 / 854$ & 0 & $1.07(0.80$ to 1.44$)$ & 0.65 & 6 & 1251 \\
\hline Diabetes & $106 / 414$ & $334 / 4460$ & 64.5 & 0.91 (0.68 to 1.21$)$ & 0.51 & 7 & 1554 \\
\hline Dyslipidaemia & $158 / 278$ & $292 / 483$ & 0 & $0.98(0.71$ to 1.36$)$ & 0.91 & 4 & 761 \\
\hline Smoking & $178 / 444$ & $337 / 1190$ & 48.3 & $0.75(0.54$ to 1.04$)$ & 0.08 & 7 & 1634 \\
\hline \multicolumn{8}{|l|}{ Clinical history: } \\
\hline $\begin{array}{l}\text { Acute coronary } \\
\text { syndrome }\end{array}$ & $48 / 206$ & $260 / 878$ & 48.5 & $1.08(0.73$ to 1.60$)$ & 0.7 & 7 & 1084 \\
\hline Stable angina & $145 / 212$ & $412 / 587$ & 47.9 & 0.95 (0.66 to 1.37$)$ & 0.78 & 5 & 799 \\
\hline Surgery & $173 / 183$ & $262 / 460$ & NE & 1.25 (0.46 to 3.37$)$ & 0.86 & 3 & 643 \\
\hline $\begin{array}{l}\text { Percutaneous } \\
\text { coronary } \\
\text { intervention }\end{array}$ & $4 / 17$ & $105 / 309$ & NE & 0.6 (0.19 to 1.88$)$ & 0.38 & 1 & 326 \\
\hline Renal impairment & $11 / 51$ & $22 / 217$ & 0 & 2.44 (1.09 to 5.44$)$ & 0.03 & 2 & 268 \\
\hline $\begin{array}{l}\text { Cerebrovascular } \\
\text { event }\end{array}$ & $113 / 129$ & $365 / 659$ & NE & $1.23(0.15$ to 9.86$)$ & 0.85 & 4 & 788 \\
\hline
\end{tabular}




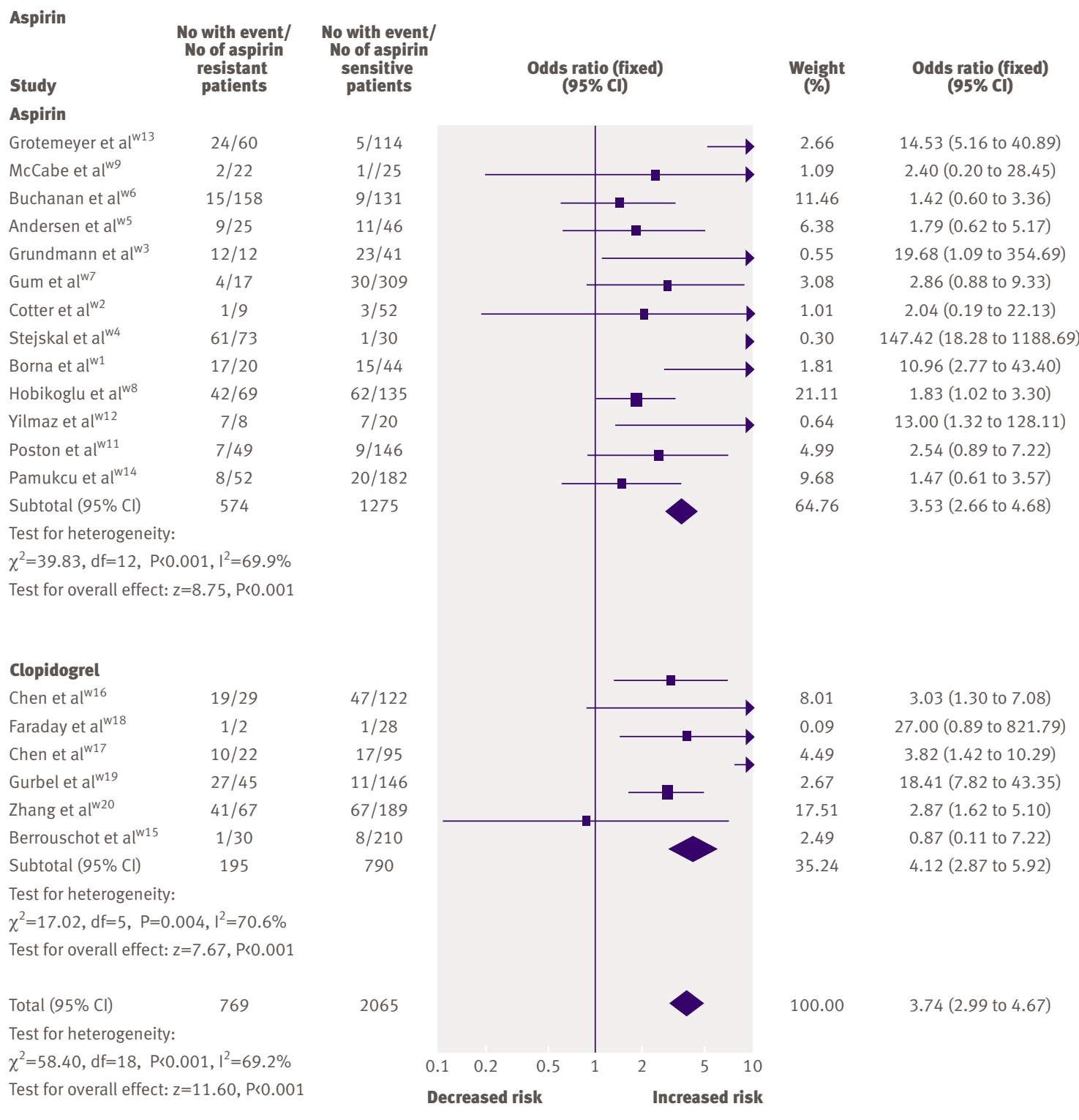

Fig 3 Risks of any cardiovascular event in aspirin resistant and aspirin sensitive patients treated with aspirin alone, and aspirin and clopidogrel (with or without an inhibitor of platelet glycoprotein Ilb/IIla)

clinical outcome, regardless of the assay used to identify patients as aspirin resistant.

We thought that the pooling of data from studies using a variety of assays for aspirin resistance was both a limitation and a strength of our study. Specifically, we rationalised that if any or all of the assays were unreliable or inaccurate in identifying patients with aspirin resistance, then any real aspirin resistant effect on clinical outcome would be undetectable. If, however, most or all of the assays did reflect some rationale and some degree of validity and sensitivity, albeit variable, then any real impact of aspirin resistance on clinical outcome should be apparent. This interpretation seemed more logical to us, since we would expect that most, if not all, investigators had considered their particular assay in good faith and with a scientific basis.
The findings of a recent review and meta-analysis of laboratory testing for aspirin resistance and clinical outcome $\mathrm{e}^{27}$ are consistent with those of our study. These investigators reported on 15 studies, 10 of which overlapped with our findings, and another three that were related to pilot or follow-up studies cited by us. Despite any discrepancy in the number of papers, the recent view also concluded that aspirin resistant patients "exhibit significantly higher risks of recurrent cardiovascular events compared with patients identified as (laboratory) aspirin sensitive. ${ }^{27}$

Platelet inhibitors such as clopidogrel and tirofiban did not provide any benefit to aspirin resistant patients. The relative effectiveness of these newer antiplatelet agents compared with aspirin has been established when their effectiveness was compared with that of aspirin alone in aspirin treated patients. For example, 
the Aspirin Trialists' Collaboration ${ }^{1}$ suggested that when used as an antiplatelet agent aspirin provides an overall $25 \%$ risk reduction. These investigators did not, however, consider aspirin resistance and how it might influence overall risk reduction. When the data reported by the Aspirin Trialists' Collaboration are reassessed with an aspirin resistance odds ratio factored in, the risk reduction in aspirin sensitive patients is likely to be greater than a 50\% risk reduction, whereas in aspirin resistant patients the risk seems to be noticeably increased. ${ }^{7}$ Thus the relative benefit of alternate antiplatelet agents needs to be reassessed prospectively against a group of aspirin sensitive patients. It is possible that the modest reported $10 \%$ better effect of alternate platelet therapies compared with aspirin alone (the overall $25 \%$ risk reduction) may well be $20 \%$ less effective when compared with the potentially greater than $50 \%$ beneficial effect of aspirin in aspirin sensitive patients. If so the current strategies for antiplatelet therapy may need to be rethought.

Our analyses also indicate that the effect of aspirin resistance on clinical outcome is applicable to the entire community of aspirin treated patients at risk of cardiovascular and cerebrovascular events. Thus the increased risk of these events in aspirin resistant patients occurs in those with stable cardiovascular disease or coronary artery disease, those who have had percutaneous coronary intervention or coronary artery bypass grafting, those undergoing other vascular procedures, and after stroke.

We found that aspirin resistant patients are at a greater risk of clinically important adverse cardiovascular events, indicating that such resistance is a biological entity that should be considered when recommending aspirin as antiplatelet therapy.

\section{Limitations of study}

We assessed 17 of the 20 included studies as of A quality - that is, they had a low risk of bias. ${ }^{\text {w1-w9 w11-w13 }}$ w15 w17-w20 These studies had an acceptable allocation of blindness, including an assessment of compliance, and a blinded strategy for measuring aspirin resistance status and clinical outcome. In three studies $^{\text {w10 w14 w20 }}$ data on compliance with blindness were insufficient or they lacked sufficient information to assess quality objectively, and these studies were ranked D. As these studies met the rest of the inclusion criteria and contained 586 patients (20\% of the total), we included them in our analysis. We can not, however, exclude the possibility that these studies may have skewed our analysis. As the overall outcomes of these studies were consistent with the overall analysis of the other 17 studies, however, we do not think this was the case.

We were unable to provide any evidence related to comorbidity and its effects on aspirin resistance. None of the included studies provided any specific information about the comorbidity characteristics between those patients who had an adverse event and those who did not in either aspirin status group. Thus the risk from being aspirin resistant seemed to be lower in men and higher in patients with a history of renal impairment. We can not provide an explanation for either at this time. The sex related difference occurred in about half of the 2930 patients, and this difference may be real. We have previously reported on sex related differences in aspirin pharmacodynamics and pharmacokinetics. ${ }^{28}$

The relation between being resistant to aspirin and having a history of renal impairment is possibly an anomaly. This relation was seen in only two studies, ${ }^{\text {w16 }}$ ${ }^{\text {w17 }}$ both from the same centre, concerning an Asian population, and representing less than $9 \%$ of the patients analysed. Thus the apparent relation between a history of renal impairment and aspirin status can not be generalised to the entire patient population. Whether this relation is unique to the Asian population is not clear but merits further exploration. We recommend therefore that future studies should consider these relations, including sex, comorbidities, and ethnicity, as these characteristics may well affect the relative risks of adverse effects in a patient population that is already at high risk.

We were unable to determine which platelet function test best identifies aspirin resistant patients. It might be argued that the platelet aggregation assays (using

Table 3 | Risks of adverse outcomes and aspirin dose response in patients who are resistant to aspirin

\begin{tabular}{|c|c|c|c|c|c|}
\hline Variable & $\begin{array}{l}\text { No of studies } \\
\quad(n=20)\end{array}$ & $\begin{array}{l}\text { No of patients } \\
\quad(n=2930)\end{array}$ & Odds ratio $(95 \% \mathrm{Cl})$ & Heterogeneity (\%) & $P$ value \\
\hline \multicolumn{6}{|l|}{ Outcome: } \\
\hline All cerebrovascular events* & 20 & 2930 & 3.85 (3.08 to 4.80$)$ & 68.3 & $<0.001$ \\
\hline Death & 4 & 728 & $5.99(2.28$ to 15.72$)$ & 5.5 & $<0.003$ \\
\hline Acute coronary syndrome & 9 & 1275 & $4.06(2.96$ to 5.56$)$ & 58.6 & $<0.001$ \\
\hline Graft failure & 3 & 420 & $4.35(2.26$ to 8.37$)$ & 28.2 & $<0.001$ \\
\hline New cerebral event & 4 & 340 & $3.78(1.25$ to 11.41$)$ & 38.1 & $<0.02$ \\
\hline \multicolumn{6}{|l|}{ Dose response: } \\
\hline $75-100 \mathrm{mg} /$ day & 3 & 228 & 21.17 (8.83 to 50.77$)$ & 74.7 & $<0.003$ \\
\hline$>100-325 \mathrm{mg} /$ day & 9 & 1,309 & $2.81(2.00$ to 3.94$)$ & 37.9 & $<0.001$ \\
\hline $500 \mathrm{mg}$ three times daily & 1 & 174 & $14.53(\mathrm{NA})$ & NA & $<0.001$ \\
\hline All doses & 12 & 1710 & 3.28 (2.39 to 4.49$)$ & 56.7 & $<0.001$ \\
\hline
\end{tabular}




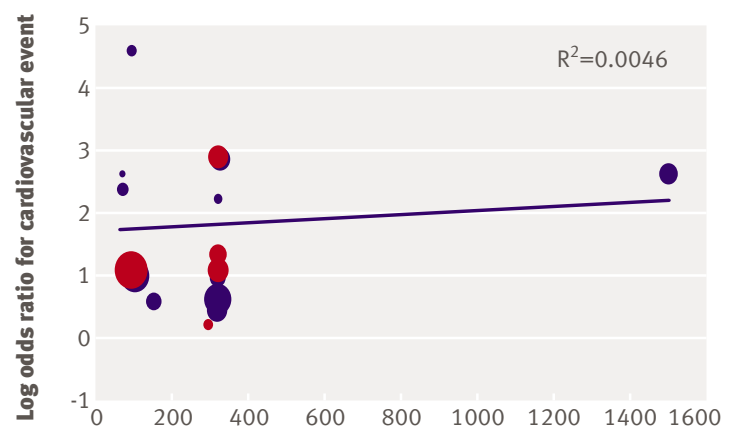

Aspirin dose (mg/day)

Fig 4 Dose related effect of aspirin on cardiovascular outcome

platelet rich plasma) are the better assays since those tests label fewer patients as aspirin resistant with less heterogeneity and a higher odds ratio than the whole blood platelet function assays, such as the platelet function analyser 100 assay. Moreover, lower heterogeneity was found with the platelet aggregation assays despite the use of a variety of agonists. It also can be argued that the greater heterogeneity seen when using the whole blood platelet function assays better reflects what is happening in vivo, as this type of assay concerns other cell types, such as inflammatory cells, that could contribute to the overall response of circulating platelets in aspirin resistant patients. The responses to particular agonists in aspirin resistant patients has yet to be elucidated. This is complicated further by the current limited understanding of the underlying mechanism of platelet function related to aspirin resistance. We previously suggested that aspirin resistance is independent of the platelet cyclo-oxygenase pathway and thromboxane $\mathrm{B}_{2}$ (thromboxane $\mathrm{A}_{2}$ ) inhibition and more related to an effect of aspirin (its salicylate moiety) on lipoxygenase dependent 12 HETE synthesis, integrin expression, and platelet adhesion in itself. ${ }^{4 w 6}$ More recent studies suggest that a pathway independent of cyclo-oxygenase-1 and cyclo-oxygenase- 2 affects aspirin resistance. ${ }^{2425}$ It is worth noting that a systematic review found a higher prevalence of aspirin resistance when using the platelet function analyser 100 assay than when using a platelet rich plasma aggregation assay, similar to our findings. ${ }^{18}$ These investigators also adjusted the prevalence of aspirin resistance on the basis of dose, study population, and aspirin resistance assay used. We did not do this, however, because we did not find any dose related effect (as with the Aspirin Trialists' Collaboration ${ }^{1}$ ) and could not exclude other variables such as comorbidity, which the previous systematic review also did not exclude. ${ }^{18}$ Therefore we suggest that this matter can only be resolved by a prospective study using a battery of aspirin resistance assays, each of which must be related to clinical outcome measures to ascertain specificity, sensitivity, and relevance. Only then can the best assay be identified.

Data were insufficient in the included studies to establish whether patients initially identified as aspirin resistant remained aspirin resistant or whether patients identified as aspirin sensitive subsequently became aspirin resistant. Either possibility could skew the overall prevalence of being aspirin resistant. Some investigators have suggested that chronic aspirin use is ineffective after about two years. ${ }^{1429}$ This possible variance is unlikely to alter the overall increased odds ratio of having an adverse event when aspirin resistant, because the clinical outcome measures in these studies were related to the initial assay for aspirin resistance.

Only a few of the studies measured a biochemical marker of compliance (for example, thromboxane $\mathrm{A}_{2}$ or thromboxane $\mathrm{B}_{2}$ ). However, most of the other investigators identified their patients as aspirin resistant or aspirin sensitive in hospital-that is, in a controlled environment after a specific procedure and before and after known aspirin treatment. Thus aspirin status measured at that time could be assured independent of non-compliance. As such it is difficult to imagine that most patients had subsequently become non-compliant, and yet a significant difference in odds ratios would remain between the initially labelled aspirin resistant patients and those who were sensitive to aspirin.

Finally, a classic funnel plot suggested a modest publication bias, since there was an absence of small patient number studies that reported on a negative or no relation between aspirin resistance and adverse clinical outcomes. We found little evidence of any publication bias using a classic funnel plot and Egger's and Macaskill modification, ${ }^{22}$ suggesting that the overall findings of our study were not skewed by publication bias.

\section{Recommendation and future directions}

Although this study shows that aspirin resistance adversely affects clinical outcomes whenever aspirin is used alone or in combination with another antiplatelet agent, several issues need resolution or clarification:

Firstly, we strongly advise that doctors continue their current practice in prescribing aspirin for chronic therapy to prevent adverse cardiovascular events as the overall risk reduction is well reported. ${ }^{1}$ We also recommend that patients are fully informed about the possible adverse effects of aspirin as it is possible that

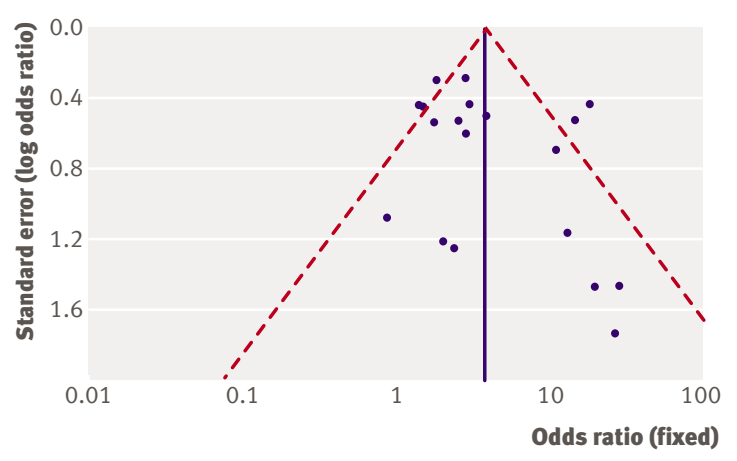

Fig 5 Funnel plot for detection of possible publication bias 


\section{WHAT IS ALREADY KNOWN ON THIS TOPIC}

Many patients with cardiovascular disease are "resistant" or non-responsive to aspirin

\section{WHAT THIS STUDY ADDS}

Aspirin resistance can be measured by a variety of tests, all of which are associated with clinically important adverse events

the currently perceived overall benefit in all aspirin treated patients (about a 25\% decrease in risk) is more likely offset by the fourfold increased risk in the $16 \%$ to $30 \%$ subpopulation of patients identified as aspirin resistant.

Studies need to be designed to determine the most useful test to identify aspirin resistance and identify alternative effective therapies for patients who are resistant to aspirin. Finally, we suggest that the term aspirin resistant is a misnomer. Aspirin non-responsiveness may be a more appropriate label until a better understanding is reached about what particular platelet function is involved.

We thank M Dang and M Kirchhoff-Dobias for translating the Chinese and Czech papers. This study was supported by discretionary funds of $\mathrm{SJ} B$ and MRB. The following authors verified our data interpretations: $K$ Andersen (Norway); J Berrouschot, K-H Grotemeyer, and H Topka (Germany); W-H Chen (China); G Hobikoǒlu and MB Yilmaz (Turkey); and R Poston (United States).

Contributors: All authors did the literature search; reviewed the abstracts, papers, and data; and reviewed the manuscript and made appropriate amendments. MRB wrote the paper. All authors are guarantors for the paper

Funding: None

Competing interests: None declared.

Ethical approval: Not required

Provenance and peer review: Not commissioned; externally peer reviewed.

1 Aspirin Trialists' Collaboration. Collaborative overview of randomized trials of antiplatelet therapy. I: Prevention of death, myocardial infarction, and stroke by prolonged antiplatelet therapy in various categories of patients. BM/ 1994:308:81-106.

2 Patrono C, García Rodríguez LA, Landolfi R, Baigent C. Low-dose aspirin for the prevention of atherothrombosis. $N$ Engl J Med 2005;353:2373-83.

3 Lee PY, Chen WH, Ng W, Cheng X, Kwok JY, Tse HF, et al. Low-dose aspirin increases aspirin resistance in patients with coronary artery disease. Am J Med 2005;118:723-7.

4 Buchanan MR, Brister SJ. Individual variation in the effects of ASA on platelet function: implications for the use of ASA clinically. Can J Cardiol 1995;11:221-7.

5 Quinn MJ, Aronow HD, Califf RM, Bhatt DL, Sapp S, Kleiman NS, et al. Aspirin dose and six-month outcome after an acute coronary syndrome. J Am Coll Cardiol 2004;43:972-8.

6 Taylor DW, Barnett HJ, Haynes RB, Ferguson GG, Sackett DL, Thorpe KE, et al. Low-dose and high-dose aspirin acetylsalicylic acid for patients undergoing carotid endarterectomy; a randomized controlled trial. ASA and Carotid Endoarterectomy (ACE) Trial Collaborators Lancet $1999 \cdot 353 \cdot 2179-84$.
7 Buchanan MR. Biological basis and clinical implications of acetylsalicylic acid resistance. Can J Cardiol 2006;22:149-51.

8 Eikelboon JW, Hirsh J, Weitz Jl, Johnson M, Yi Q, Yusef S. Aspirinresistant thromboxane biosynthesis and risk of myocardial infarction, stroke, or cardiovascular death in patients at high risk for cardiovascular events. Circulation 2002;105:1650-5.

9 Patrono C. Aspirin resistance: definition, mechanisms and clinical read-outs. J Thromb Haemost 2003;1:1710-3.

10 Hennekens $\mathrm{CH}$, Schor K, Weisman S, Fitzgerald GA. Semantic complexity and aspirin resistance. Circulation 2004:110:1706-8.

11 Cattaneo M. Aspirin and clopidogrel: efficacy, safety, and the issue of drug resistance. Aterioscler Throm Vasc Biol 2004;24:1980-7.

12 Kuliczkowski W, Halawa B, Korolko B, Mazurek W. Aspirin resistance in ischemic heart disease. Kardiol Pol 2005; 62:14-9.

13 Angiolillo DJ, Fernandez-Ortiz A, Bernardo E, Ramírez C, Sabaté M, jimenez-Quevedo $\mathrm{P}$, et al. Influence of aspirin resistance on platelet function profiles in patients on long-term aspirin and clopidogrel after percutaneous coronary intervention. Am J Cardiol 2006;97:38-43.

14 Hegason CM, Bolin KM, Hoff JA, Winkler SR, Manget A, Tortorice KL, et al. Development of aspirin resistance in persons with previous ischemic stroke. Stroke 1994;25:2331-6.

15 Kawasaki T, Ozeki Y, Igawa T, Kambayashi J-I. Increased platelet sensitivity to collagen in individuals resistant to low-dose aspirin. Stroke 2000;31:591-6.

16 Macchi L, Christianes L, Brabant S, Sorel N, Allal J, Mauco, et al. Resistance to aspirin is associated with platelet sensitivity to adenosine diphosphate. Thromb Res 2002;107:45-9.

17 Zimmerman N, Wenk A, Kim U, Kienzle P, Weber AA, Gams E, et al. Functional and biochemical evaluation of platelet aspirin resistance after coronary artery bypass surgery. Circulation 2003;108:542-7.

18 Hovens MMC, Snoep JD, Eikenboom ICI, van der Bom JG, Mertens BIA, Huisman MV. Prevalence of persistent platelet reactivity despite use of aspirin: a systematic review. Am Heart J 2007;153:175-81.

19 Veichtbauer: MiMa: An S-plus/R function to fit meta-analytic mixedrandom and fixed effects models [computer and software]. www. wvbauer.com.

20 Egger M, Davey Smith G, Schneider M, Minder C. Bias in meta-analysis detected by a simple, graphical test. BMJ 1997;315:629-34

21 Stroup DF, Berlin JA, Morton SC, Olkin I, Williamson CD, Rennie D, et al. Meta-analysis of observational studies in epidemiology. A proposal for reporting. JAMA 2000;283:2008-12.

22 Macaskill P, Walter SD, Irwig L. A comparison of methods to detect publication bias in meta-analysis. Stat Med 2001;20:641-54

23 Van Ryn McKenna J, Buchanan MR. Relative effects of fluriprofen on platelet 12-hydroxyeicostetraenoic acid and thromboxane $\mathrm{A}_{2}$ production: influence on collagen-induced platelet aggregation and adhesion. Prost Leuko Med 1989;36:171-4.

24 Abaci A, Caliskan M, Bayram F, Yilmaz Y, Cetin M, Unal A, et al. A new definition of aspirin non-responsiveness by Platelet Function Analyser-100 ${ }^{\mathrm{TM}}$ and its predictors. Platelets 2006;17:7-13.

25 Heindl B, Becker BF. Aspirin, but not the more selective cyclooxygenase (COX)-2 inhibitors meloxicam and SC 58125, aggravates post ischemic cardiac dysfunction, independent of COX function. Nauyn-Schmiedeberg's Arch Pharmacol 2001;363:233-40.

26 Frelinger AL III, Furman MI, Linden MD, Li Y, Fox ML, Barnard MR, et al. Residual arachidonic acid-induced platelet activation via an adenosine diphosphate-dependent but cyclooxygenase-1- and cyclooxygenase-2-independent pathway: a 700-patient study of aspirin resistance. Circulation 2006;113:2888-96.

27 Snoep JD, Hovens MMC, Eikenboom JCJ, van der Bom JG, Huisan MV. Association of laboratory-defined aspirin resistance with a higher risk of recurrent cardiovascular events. A systematic review and metaanalysis. Arch Intern Med 2007;167:1593-9.

28 Buchanan MR, Rischke JA, Butt R, Turpie AGG, Hirsh J, Rosenfeld J. The sex-related differences in platelet function and aspirin pharmacokinetics in rabbits and man. Thromb Res 1983:29:469-70.

29 Helgason CM, Tortorice KL, Winkler SR, Penney DW, Schiler JJ, McClelland TJ, et al. ASA response and failure in cerebral infarction. Stroke 1993;25:345-50.

Accepted: 6 November 2007 